\title{
Presheaf (of abelian groups) on a topological space
}

\author{
Open Mathematics Collaboration*广
}

February 17, 2021

\begin{abstract}
PRESHEAF and its underlying definitions are presented in this white paper (knowledge base).
\end{abstract}

keywords: sheaf theory, abelian group, topological space, knowledge base

The most updated version of this white paper is available at https://osf .io/2y5s4/download

*All authors with their affiliations appear at the end of this white paper.

†Corresponding author: mplobo@uft.edu.br|Open Mathematics Collaboration 


\section{Definition}

1. Presheaf (of abelian groups) on a topological space $X$

$$
\begin{gathered}
f: U \rightarrow A(U) \\
r_{U, V}: A(V) \rightarrow A(U)
\end{gathered}
$$

(a) $r_{U, U}=1$

(b) $r_{U, V} r_{V, W}=r_{U, W}$ when $U \subset V \subset W$

$f, r_{U, V}:=$ functions

$U, V:=$ open sets

$X:=$ topological space

$U \subset V$

$U \subset X$

$A:=$ presheaf

$A(U):=$ abelian group

$r_{U, V} \equiv$ homomorphism (restriction)

$[1]$ 


\section{Prerequisites}

2. Ordered pair

$$
(a, b)
$$

$a:=$ first coordinate

$b:=$ second coordinate

$[2]$

3. Cartesian product

$$
A \times B=\{(a, b) \mid a \in A, b \in B\}
$$

$A, B:=$ sets

$A \times B:=$ Cartesian product

$[2]$

4. Binary operation

$$
\star: S \times S \rightarrow S
$$

$S:=$ set

$S \times S:=$ Cartesian product

$[3]$

5. Group

$$
(G, \star)
$$
(a) Associativity: $\forall x, y, z \in G,(x \star y) \star z=x \star(y \star z)$
(b) Identity: $\exists e \in G: \forall x \in G, e \star x=x \star e=x$
(c) Inverse: $\forall x \in G \exists y \in G: x \star y=y \star x=e$
$G:=$ set
$\star:=$ binary operation 
[3]

6. Abelian group

$$
\begin{gathered}
G_{b} \\
\forall g_{1}, g_{2} \in G_{b}, g_{1} g_{2}=g_{2} g_{1}
\end{gathered}
$$

$G_{b}:=\operatorname{group}$

[4]

7. Open interval

$$
(a, b)=\{x \in X \mid a<x<b\}
$$

$X:=$ set

[5]

8. Open set

$$
X:=\text { open set in } Y
$$
(a) $X \subseteq Y$
(b) $\forall x \in X, \exists(a, b): x \in(a, b),(a, b) \subseteq X$
$X, Y:=$ sets
$(a, b):=$ open interval
[5]

9. Arbitrary Union

$X:=$ collection of sets

$\bigcup X:=\{y \mid \exists Y \in X, y \in Y\}$

[5] 


\section{Arbitrary Intersection}

\section{$\bigcap X$}

$X:=$ collection of sets

$\cap X:=\{y \mid \forall Y \in X, y \in Y\}$

[5]

11. Topology on $S$

$S:=$ set

$\mathcal{T}:=$ collection of open subsets of $S$

$X, Y:=$ collection of sets

$\bigcup X:=$ arbitrary union

$\cap Y:=$ arbitrary intersection

(a) $\varnothing, S \in \mathcal{T}$

(b) $(X \subseteq \mathcal{T}) \rightarrow(\bigcup X \in \mathcal{T})$

[ $\mathcal{T}$ is closed under arbitrary unions]

(c) $(Y \subseteq \mathcal{T}, Y$ finite $) \rightarrow(\cap Y \in \mathcal{T})$

$[\mathcal{T}$ is closed under finite intersections]

$[5,6]$

\section{Topological space}

$S:=$ set

$\mathcal{T}:=$ topology on $S$

$[5,6]$ 


\section{Homomorphism}

$$
f^{h}
$$

(a) $f^{h}: G \rightarrow H$

$$
\forall x, y \in G: f^{h}(x * y)=f^{h}(x) \circ f^{h}(y)
$$

$f^{h}:=$ function

$G, H:=$ sets

$*, \circ:=$ binary operations

$(G, *),(H, \circ):=$ groups

$[3,4,7]$

\section{Open Invitation}

Review, add content, and co-author this white paper [8,9].

Join the Open Mathematics Collaboration.

Send your contribution to mplobo@uft.edu.br.

\section{Open Science}

The latex file for this white paper together with other supplementary files are available in [10].

\section{Ethical conduct of research}

This original work was pre-registered under the OSF Preprints [11], please cite it accordingly [12]. This will ensure that researches are conducted with integrity and intellectual honesty at all times and by all means. 


\section{Acknowledgement}

+ Center for Open Science

https://cos.io

+ Open Science Framework

https://osf .io

\section{References}

[1] Bredon, Glen E. Sheaf theory. Vol. 170. Springer Science \& Business Media, 2012.

https://books.google.com/books?id=JFXSBwAAQBAJ

[2] Velleman, Daniel J. How to prove it: A structured approach. Cambridge University Press, 2019.

https://books.google.com/books?id=sXt-ROLLNHcC

[3] Warner, Steve. Abstract Algebra for Beginners. GET 800, 2018.

https://books.google.com/books?id=UFleyAEACAAJ

[4] Dummit, David Steven, and Richard M. Foote. Abstract Algebra. Vol. 3. Hoboken: Wiley, 2004.

https://books.google.com/books?id=znzJygAACAAJ

[5] Warner, Steve. Topology for Beginners. GET 800, 2019. https://books.google.com/books?id=pNAvxQEACAAJ

[6] Munkres, James R. Elements of algebraic topology. CRC press, 2018. https://books.google.com/books?id=-mdQDwAAQBAJ

[7] Rotman, Joseph J. A first course in abstract algebra. Pearson College Division, 2000.

https://books.google.com/books?id=ctEZAQAAIAAJ 
[8] Lobo, Matheus P. "Microarticles." OSF Preprints, 28 Oct. 2019. https://doi.org/10.31219/osf.io/ejrct

[9] Lobo, Matheus P. "Simple Guidelines for Authors: Open Journal of Mathematics and Physics." OSF Preprints, 15 Nov. 2019.

https://doi.org/10.31219/osf .io/fk836

[10] Lobo, Matheus P. "Open Journal of Mathematics and Physics (OJMP)." OSF, 21 Apr. 2020.

https://doi.org/10.17605/osf .io/6hzyp

https://osf.io/6hzyp/files

[11] COS. Open Science Framework. https://osf .io

[12] Lobo, Matheus P. "Presheaf (of Abelian Groups) on a Topological Space." OSF Preprints, 3 Feb. 2021.

https://doi.org/10.31219/osf .io/2y5s4

\section{The Open Mathematics Collaboration}

Matheus Pereira Lobo (lead author, mplobo@uft.edu.br) $)^{1,2}$ https://orcid.org/0000-0003-4554-1372

${ }^{1}$ Federal University of Tocantins (Brazil)

${ }^{2}$ Universidade Aberta (UAb, Portugal) 